\title{
Depressão em oncologia: Um olhar da psicologia
}

\author{
Depression in oncology: A view of psychology
}

Itala Villaça Duarte ${ }^{[a]}$, Aline Mariana Rodicz ${ }^{[b]}$

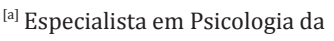
Saúde pela Faculdade Pequeno Príncipe e em Cancerologia com ênfase em Psicologia pelo Hospital Erasto Gaertner, psicóloga clínica e hospitalar do Hospital Erasto Gaertner e Preceptora da Residência em Cancerologia com ênfase em Psicologia nesta mesma instituição (HEG), Curitiba, PR Brasil, e-mail:

itala.duarte@hotmail.com

${ }^{[b]}$ Psicóloga, especialista em Psicologia Hospitalar com ênfase em Oncologia pelo Hospital Erasto Gaertner (HEG), psicóloga clínica do Serviço de Psicologia da mesma instituição (HEG), Curitiba, PR - Brasil, e-mail: alinemariana@lpcc.org.br
}

Recebido: 30/01/2012 Received: 01/30/2012

Aprovado: 02/05/2012 Approved: 05/02/2012

\section{Resumo}

A depressão representa um problema de saúde pública e pode aparecer associada a uma doença clínica como, por exemplo, o câncer. A depressão na oncologia tem sido cada vez mais relatada, no entanto, seu diagnóstico ainda representa um desafio aos profissionais devido às semelhanças da depressão com os sintomas da doença oncológica, os efeitos adversos que a quimioterapia possivelmente causa nos pacientes e as repercussões emocionais vivenciadas pelo processo de adoecimento. Com isso, há probabilidade de o diagnóstico ser realizado com imprecisão e o manejo terapêutico muitas vezes se torna inadequado. A proposta mais apropriada seria aquela que contempla o tratamento psiquiátrico e farmacológico em conjunto com o acompanhamento psicológico. Em relação a esse acompanhamento, especificamente, é por meio da relação terapeuta-paciente que se torna possível favorecer ao paciente a expressão de experiências e sentimentos, permitindo que sejam assimilados, compreendidos e vivenciados, e que, consequentemente, o paciente possa encontrar formas adaptativas e conciliatórias para aderir e continuar o tratamento oncológico.

Palavras-chave: Depressão. Oncologia. Psicologia.

\section{Abstract}

Depression represents a public health problem and may be associated with a clinical disease such as cancer. Depression in oncology has been increasingly reported, but its diagnosis is still a challenge for professionals due to the similarities between depression and the symptoms of cancer, side effects that chemotherapy can cause in the patients and the emotional consequences experienced by the process of illness. In this sense, there is a chance that the diagnosis is performed with imprecision and therapeutic management often becomes inadequate. The appropriate choice would be one that includes psychiatric and pharmacological treatment in conjunction with counseling. In relation to this monitoring, specifically, it is through the therapist-patient relationship that it may be possible to encourage the expression of the patient's experiences and feelings, allowing them to be assimilated, understood and experienced. And that, consequently, the patient may find adaptive and conciliatory ways to join and continue cancer treatment.

Keywords: Depression. Oncology. Psychology. 


\section{Introduç̃̃o}

0 adoecimento, seja por uma doença crônica ou aguda, envolve algum tipo de debilidade que necessitará de cuidados e tratamento, que, por sua vez, compreendem desde consultas médicas e uso de medicamentos até internamentos e cirurgias. Esse adoecer, então, reflete a fragilidade do corpo humano, trazendo à consciência a percepção da finitude e, em decorrência disso, repercussões em diversos âmbitos como: social, emocional, familiar e profissional, de acordo com a estrutura psíquica do indivíduo, bem como a gravidade e/ou necessidade do caso.

Essas repercussões podem ser intensificadas quando o adoecimento ocorre por alguma neoplasia, tendo em vista os estigmas e representações sociais em relação a essa doença e tratamentos. Paciente e familiares se deparam com a perspectiva econômico-sociocultural e espiritual da cronicidade e mortalidade dessa doença, a qual deverá ser tratada como se combatendo a um inimigo, com armas agressivas, ou seja, os tratamentos por vezes invasivos, causadores de dor e sofrimento físico e emocional. Diante desse contexto, deve-se considerar que a doença exerce uma ameaça sobre a existência humana individual e familiar, fazendo com que, em algum momento do processo de diagnóstico e tratamento, seja acompanhada de perturbações emocionais, incluindo a depressão (Trancas, Cardoso, Luengo, Vieira, \& Reis, 2010).

A depressão como comorbidade psiquiátrica associada a alguma doença clínica é passível de ser entendida como uma dificuldade de adaptação que o paciente apresenta para enfrentar o adoecimento (Gaspar, 2011). Alguns fatores influenciam o aparecimento e a manutenção dessa comorbidade, tais como a hospitalização prolongada, medicamentos, tratamento invasivo, metástase, dor crônica, prognóstico reservado e recursos psíquicos de enfrentamento disponíveis no paciente (Baptista, Santos, \& Dias, 2006; Bottino, Fráguas, \& Gattaz, 2009). Assim, o diagnóstico de depressão no contexto hospitalar torna-se um desafio para os profissionais tanto pela superposição dos sintomas com a doença oncológica, pelos efeitos adversos que a quimioterapia poderá causar, bem como pelas repercussões emocionais vivenciadas no processo de adoecimento. Deve-se, então, avaliar a gravidade e a duração dos sintomas para um diagnóstico diferencial e uma conduta terapêutica adequada que possa abranger o tratamento farmacológico, psiquiátrico e psicológico.

A intervenção psicológica deve contemplar a anamnese, considerando aspectos econômicos, biopsicossociais e espirituais, o acompanhamento do caso e trocas multiprofissionais. 0 acompanhamento psicológico baseia-se no relacionamento terapêutico entre terapeuta-paciente como forma de crescimento deste último. Nesta relação, o terapeuta visa proporcionar condições favoráveis que possibilitem ao paciente entrar em contato com suas potencialidades internas e (re)começar a traçar caminhos a serem percorridos. Nessas condições tem-se a autenticidade, a empatia, a congruência, a aceitação e a escuta atenta e interessada do terapeuta. Essa vivência pode possibilitar ao paciente o reconhecimento e a ressignificação das experiências a partir da sua tendência atualizante e campo fenomenal proporcionando uma adaptação à situação de adoecimento.

Essas questões serão abordadas ao longo do presente artigo, no qual se buscará um estudo acerca da depressão na oncologia e os cuidados terapêuticos, propondo uma reflexão e a (re)construção de um olhar para esta realidade, comum no contexto de adoecimento e hospitalização.

\section{Adoecimento oncológico}

No cenário de adoecimento e tratamento oncológico, em cada fase do câncer — desde pré-diagnóstico, diagnóstico, tratamento, pós-tratamento, progressão da doença, recidiva até cuidados paliativos-, uma ebulição de sentimentos aparece, sendo frequente ansiedade, medo, angústia, tristeza, desespero, desesperança, e preocupação em relação à morte e incertezas quanto ao futuro causando sofrimento psíquico e emocional, além de influenciar nos aspectos orgânicos (Liberato \& Carvalho, 2008).

Alguns fatores característicos desse momento chegam a repercutir emocionalmente no paciente, os quais são: presença da doença avançada (metástase) tendo prognóstico reservado; uso de alguns quimioterápicos (interferon, vimblastina, vincristina, tamoxifeno); dor crônica e não controlada; prejuízos nas habilidades funcionais e vocacionais; perda da individualidade e autonomia pela hospitalização; longo período de internação 
afetando o convívio social e familiar; dependência dos profissionais de saúde e familiares; sítios da doença e questões relacionadas à imagem corporal (mutilações, alopecia); mudanças de rotina devido à constante frequência ao hospital para consulta, exames e internamentos; e mudanças na rotina e reestruturação de papéis sociais e familiares que não foram escolhidos e sim impostos pela fatalidade do adoecimento (Baptista et al., 2006; Bottino et al., 2009).

Desta maneira, observa-se a diversidade de questões inerentes ao adoecimento e tratamento que influenciam a realidade emocional com intensidade e frequência variadas de acordo com a estrutura de personalidade, perfil afetivo e os recursos de enfrentamento de cada paciente. A partir dessa perspectiva, considera-se que:

[...] muitas das alterações emocionais que acometem aos pacientes oncológicos não são propriamente reações patológicas e sim reações humanas pelos conflitos que emergem e que são chamadas formalmente de reações de ajustamento, desencadeadas por situações de crise como é a condição de quem adoece por câncer (Gaspar, 2011, p. 106).

Essas reações de ajustamento poderiam ser consideradas apenas como uma resposta ao impacto do diagnóstico e tratamento oncológico, ou seja, um transtorno específico do humor que se encontra no meio do caminho entre o normal e o patológico, sendo este último representado por um transtorno de maior gravidade psiquiátrica, como a depressão (Botega, 2006 citado por Gaspar, 2011).

Portanto, algum grau de tristeza (humor deprimido) é esperado e necessário para o enfrentamento e reestruturação psíquica do indivíduo (Juver \& Verçosa, 2008). Contudo, nem sempre essa reestruturação emocional ocorre e a tristeza se apresenta como fator preponderante conjuntamente com outras características como apatia ou irritabilidade, bem como o isolamento social e sentimentos de autodesvalorização, entre outros. Assim, Gaspar (2011, p. 106) afirma que:

Quando o impacto emocional relacionado ao diagnóstico e ao tratamento do câncer é persistente, ou quando os recursos emocionais dos pacientes são insuficientes para lidar com a situação, o desfecho poderá ser o sofrimento emocional.

Com isso, deve-se avaliar a cronicidade, gravidade e persistência dos sintomas apresentados e o tempo de duração para um diagnóstico preciso e diferencial, além de uma proposta terapêutica adequada a cada paciente.

A proposta de trabalho multiprofissional ou interdisciplinar se torna imprescindível para entender se essa tristeza é reativa à situação vivenciada ou se existe outra patologia presente, como a depressão, haja vista que alguns sintomas se assemelham. Portanto, é necessária uma avaliação minuciosa visando a um diagnóstico diferencial e uma proposta terapêutica apropriada que englobe tanto tratamento farmacológico e psiquiátrico quanto acompanhamento psicológico.

\section{Depressão}

A depressão se tornou um termo culturalmente utilizado para assinalar um estado de humor, a tristeza, tornando seu uso coloquial. Clinicamente, a depressão é considerada um transtorno psiquiátrico, atualmente com alta prevalência na população em geral e associada a outras doenças. Está relacionada às questões afetivas ou de humor, sendo caracterizada por uma alteração psíquica e orgânica global, com consequentes maneiras diferentes de valorizar e vivenciar a realidade, como planejar a vida. Sua apresentação dá-se por um conjunto de transformações em diversos âmbitos físicos e emocionais. (Juver \& Verçosa, 2008).

Essas possíveis alterações presentes no quadro depressivo são encontradas nas esferas: afetiva com humor deprimido, apatia ou irritabilidade; psicomotora tendo como característica agitação ou lentificação física; cognitiva em razão do déficit de atenção, concentração e memória; instintiva e neurovegetativa apresentando fadiga, desânimo, alteração do sono e apetite; ideativa caracterizada por pessimismo, ideias de arrependimento, fracasso e culpa desesperança e suicídio; autodesvalorização com sentimento de baixa autoestima, sentimento de insuficiência e inutilidade; e, por fim, sintomas psicóticos devido à presença de delírios e alucinações (Juver \& Verçosa, 2008). 
"Essas alterações quase sempre levam ao comprometimento do desempenho interpessoal, social e ocupacional, além disso, há uma experiência subjetiva de grande sofrimento" (Kaplan \& Sadock, 2007 citados por Gaspar, 2011, p. 108). Devido à experiência subjetiva de cada indivíduo, nem sempre há a presença de todos esses sintomas característicos da depressão, sua apresentação varia conforme a estrutura de personalidade e os recursos físicos e psíquicos adaptativos disponíveis.

As razões para o surgimento da depressão também se diferenciam por outros fatores como: vulnerabilidade genética e social, doenças clínicas ou uso de medicamentos que favorecem o aparecimento da depressão. Além desses critérios, um estresse mais intenso ou uma desorganização emocional podem ser também desencadeadores de uma crise depressiva.

Diante destas questões, considera-se que a presença de doenças clínicas seja um critério significativo para o surgimento da depressão, bem como um fator desencadeador ou intensificador. As razões pelas quais a depressão se apresenta junto a uma doença clínica equivalem ao processo de adoecimento, hospitalização, o uso de medicamentos e as repercussões emocionais, sociais e familiares envolvidas nesta situação. Dentre as doenças clínicas, o câncer tem, em relação à depressão, uma prevalência de 22 a 29\% (Bottino et al., 2009).

Com essa prevalência significativa entre depressão e oncologia, é necessária a valorização de uma conduta terapêutica abrangendo não apenas seus aspectos físicos, como também emocionais. A atuação de uma equipe multiprofissional e interdisciplinar se faz imprescindível para compreender o indivíduo, a doença e a relação entre ambos a partir de uma perspectiva integral.

\section{Depressão e oncologia: os cuidados da Psicologia}

A depressão como comorbidade psiquiátrica associada a algum tipo de neoplasia é passível ser entendida como uma forma de reação desajustada ou uma dificuldade de adaptação que o paciente apresenta para enfrentar a situação de adoecimento e as repercussões do diagnóstico e tratamento. É necessário, portanto, conhecer o funcionamento psíquico e emocional deste indivíduo, principalmente frente a situações de adversidades, para compreender suas reações e assim ter melhores condições de diferenciar os sintomas da depressão dos sintomas do câncer e/ou dos efeitos tóxicos secundários resultantes do tratamento quimioterápico (Gaspar, 2011).

Além disso, deve-se levar em consideração também a idade do paciente e seus planos de vida, seus antecedentes pessoais e familiares, aspectos econômicos, sociais e culturais, bem como se existe rede de apoio. Outros aspectos importantes de serem avaliados como fatores de risco em doentes oncológicos são: isolamento social, perdas recentes, tendência ao pessimismo, presença de dor, pressões socioeconômicas, abuso de substâncias, história de perturbações do humor, como a depressão, ou tentativas de suicídio (Trancas et al., 2010).

No que concerne ao adoecimento oncológico, é importante também compreender qual a representação da doença para o paciente, o tempo do diagnóstico e evolução da doença, tipo e local do câncer, local de tratamento (enfermaria ou ambulatório) e o impacto significativo da multimodalidade de tratamentos - cirurgia, radioterapia, quimioterapia - administrados isoladamente ou de forma associada. Em relação ao tratamento, ressalta-se o uso de quimioterápicos devido às interações medicamentosas e os possíveis efeitos adversos causados pelos mesmos, dentre eles destacam-se interferon, vimblastina, vincristina e tamoxifeno (Baptista et al., 2006; Bottino et al., 2009; Trancas et al., 2010).

A compreensão das influências destes fatores predisponentes no modo de enfrentamento do paciente tem chances de oferecer aos profissionais de saúde condições de realizar um diagnóstico mais preciso de depressão e tratamento adequado. 0 diagnóstico correto, então, promove uma conduta terapêutica adequada e melhora dos sintomas no paciente. No entanto, nem sempre esses fatores são considerados e avaliados. Vê-se na literatura que isso ocorre pelo fato da depressão, muitas vezes, ser vista como inevitável no decurso de uma doença somática e constituir uma resposta natural e esperada, por isso não sendo necessário um diagnóstico formal e uma terapêutica especializada; outro aspecto apontado seria o tempo disponibilizado nas consultas pelos profissionais de saúde ser insuficiente e pela dificuldade que muitos deles têm de falar e escutar sobre as emoções dos pacientes (Trancas et al., 2010).

Em decorrência dessas questões, há alta incidência da depressão, sendo "subdiagnosticada e, 
quando corretamente diagnosticada, é muitas vezes tratada de forma inadequada, com subdoses de medicamentos e manutenção de sintomas residuais, que comprometem a evolução clínica dos pacientes" (Teng, Humes, \& Demetrio, 2005, p. 150). De acordo com Trancas et al. (2010), em média, 25 a $50 \%$ dos diagnósticos de depressões passam despercebidos pelos profissionais de saúde e, ainda assim, quando são detectados, muitas vezes não são considerados no processo clínico.

Quando isso acontece, consequências físicas e psicológicas podem surgir no paciente como uma piora do quadro clínico em relação à depressão, ao câncer, ou até mesmo dos dois. Em virtude disso, o paciente adere menos ao tratamento proposto e às orientações médicas e, como consequência, são necessárias internações mais prolongadas. Este contexto influencia tanto no prognóstico como na qualidade de vida do paciente e seus familiares, repercutindo numa maior morbidade e risco de mortalidade.

Diante disso, uma avaliação do paciente em que sejam considerados os aspectos econômicos, biopsicossociais e espirituais poderia facilitar na proposta e eficácia do tratamento. Assim como a avaliação, se faz pertinente um tratamento integral que comporte concomitantemente o tratamento farmacológico, psiquiátrico e psicológico.

Em relação ao tratamento farmacológico e psiquiátrico, a literatura traz o uso de medicamentos antidepressivos, estabilizadores do humor ou antipsicóticos, em que as doses e a escolha do fármaco sejam realizadas de forma cautelosa, considerando fatores como as características individuais, o tipo de transtorno envolvido e a intensidade dos sintomas devido à possibilidade de ocorrência de efeitos colaterais (Pasquini \& Biondi, 2007, citados por Graner, Cezar \& Teng, 2008; Teng et al. 2005). 0 tratamento com esses antidepressivos tem chances de ser efetivo e melhorar a adesão aos tratamentos do câncer, uma vez que pode proporcionar a redução de alguns efeitos adversos, como náusea, dor e fadiga (Bottino et al., 2009). No entanto, o uso de antidepressivos também chega a causar nos pacientes efeitos adversos que devem ser observados para que, caso seja necessário e possível, um reajuste do medicamento seja realizado com objetivo de adequação terapêutica.

O acompanhamento psicológico enquanto opção de tratamento para a depressão ocorre por meio de uma avaliação psicológica inicial e realização de psicoterapia individual ou em grupo associadas ao tratamento medicamentoso. A intervenção psicológica objetiva promover, através da relação terapêutica, um espaço para a livre expressão dos sentimentos e percepções do paciente, dando assim lugar à subjetividade, para que ele possa buscar estratégias de enfrentamento mais adaptativas para a situação vivenciada de adoecimento e tratamentos.

A avaliação psicológica inicial, chamada também de anamnese, tem como finalidade conhecer o paciente e sua rede de apoio, bem como, de acordo com Gaspar (2011), reconhecer fatores de risco que possam agravar o quadro emocional atual. Portanto, é necessário um levantamento de dados em relação à vida do paciente e seus relacionamentos interpessoais, contexto familiar e questões socioculturais anteriores à doença e atualmente. Outros aspectos a serem avaliados referem-se aos antecedentes pessoais e familiares de câncer, depressão ou outros transtornos psiquiátricos existentes, além de uma investigação da presença de sintomas depressivos e a exclusão de outros transtornos psiquiátricos no paciente. A observação da dinâmica do paciente, abrangendo os aspectos orgânicos, psicológicos, sociais e seus recursos de enfrentamento, além do entendimento das capacidades e limites do terapeuta e a compreensão das possibilidades do processo terapêutico, tornam-se pertinentes para um acompanhamento individualizado e integral.

A partir dessa avaliação, quando acordado com o paciente, é possível a continuidade do trabalho psicológico através do acompanhamento sequencial. Esse acompanhamento se dá essencialmente pela relação entre terapeuta e paciente, sendo esse encontro humano e genuíno "uma atividade colaborativa entre paciente e o terapeuta, que visa à descoberta de recursos criativos, que se comportam como facilitadores internos e externos" (Liberato \& Carvalho, 2008, p. 341). Essa relação pode proporcionar ao paciente segurança e confiança, formando assim uma aliança terapêutica que possibilita a ele a expressão de forma autêntica e subjetiva, de acordo com sua disponibilidade e recursos psíquicos internos.

"Ao terapeuta cabe apenas criar as condições para que, através daquela relação particular, o indivíduo possa reorganizar-se e reencontrar a sua própria direção" (Santos, 2004, p. 21). Para isso, o terapeuta deve realizar acolhimento e psicoterapia de 
apoio criando condições inter-relacionais pautadas na autenticidade, empatia, congruência e, de acordo com o quadro de referência interno do paciente, procurar compreender a consciência vivencial de sua experiência em relação a si e ao mundo.

Com isso, visa-se promover o reconhecimento e a ressignificação do momento, sendo, portanto, terapêutico e facilitador do crescimento do paciente. E, como instrumento de trabalho, ter uma escuta minuciosa e ativa dos conteúdos apresentados e emoções vivenciadas.

Por escuta ativa entende-se um conjunto de atitudes que o psicólogo precisa ter para propiciar. Essas atitudes são desde proporcionar um ambiente físico com privacidade, conforto e proximidade interpessoal adequada até respirar momentos de silêncio e de choro, passando por formas mais abrangentes e delicadas de realizar as perguntas, além de compreender o conteúdo e a conotação da mensagem a partir, também da observação de posturas, gestos e tom de voz (Botega, 2006 citado por Gaspar, 2011, p. 106).

Assim, é pelo saber ouvir efetivamente que o psicólogo compreende qual o tipo de sofrimento que habita o paciente, de acordo com seu campo fenomenal. Ressalta-se, então, a importância de conhecer junto ao paciente, por sua perspectiva e experiência, quais são as suas principais aflições (Gaspar, 2011). E, então, a atuação do psicólogo deve se adequar à demanda que lhe é apresentada, "[...] considerando-se a particularidade de cada pessoa atendida, do tipo de câncer que incide na unicidade de um corpo psicossomático e de seu contexto vivencial" (Peçanha, 2008, p. 216).

Ao vislumbrar cada paciente como único e singular, o terapeuta poderá traçar intervenções que possam ser efetivas favorecendo ao paciente o enfrentamento da doença e dos tratamentos. Quanto ao enfrentamento, compreende-se "[...] como um processo multidimensional de mobilização do indivíduo em termos emocionais, comportamentais e cognitivos visando à adaptação a uma situação de perigo ou de desafio" (Peçanha, 2008, p. 210). Considera-se, portanto, possibilidades de lidar com uma realidade que se apresenta como ameaçadora, variando conforme a relação estabelecida entre o indivíduo e o contexto vivenciado. Em virtude disso, “[...] não há estratégia per se melhor ou pior; o que importa é avaliar a funcionalidade no contexto da pessoa e sua doença" (Peçanha, 2008, p. 210).

Quando os recursos de enfrentamento disponíveis se apresentam como funcionais, portanto, facilitadores para a situação, são capazes de possibilitar ao paciente uma abertura à experiência em um processo de vir-a-ser. Nesse processo do paciente construir seu próprio caminho, o terapeuta faz o papel de guia e acompanhante, ouvinte atento das histórias, sofrimento, emoções e percepções. 0 terapeuta como acompanhante, com sua escuta interessada, permite que o paciente se sinta percebido, considerado e acolhido, possibilitando que, por espelhamento, tenha maior interesse por si mesmo, abrindo espaço para se perceber e sentir, experimentar novas maneiras de compreender e vivenciar o momento (Juliano, 1999).

No entanto, um paciente oncológico com depressão, mesmo durante o acompanhamento psicológico, por vezes apresenta oscilação de humor num processo que pode ser compreendido como um ciclo de evolução-involução. Questões psíquicas e emocionais juntamente com aspectos físicos e sociais relacionados ao adoecimento e ao tratamento, além de características da personalidade, se inter-relacionam e interferem na maneira como o paciente vivenciará os momentos.

Em relação a esse aspecto, a autora Juliano (1999, p. 22) traz que mesmo "a cada ciclo de involução se segue um ciclo de evolução. Essa alternância nada mais é do que as duas faces de um mesmo fenômeno: o crescimento". Compreendendo dessa forma, o terapeuta consegue ter uma visão mais flexível tanto do paciente quanto de sua maneira de vivenciar a experiência. A partir disso, o terapeuta se abre para possibilidades percebendo e valorizando de forma adequada e eficaz os recursos que o paciente disponibiliza, fazendo com que esses momentos críticos sejam plenos de possibilidades de mudanças.

E no processo de possíveis mudanças, além de uma atuação em equipe multidisciplinar e interdisciplinar, os familiares também estão implicados e envolvidos na realidade vivencial do paciente, sendo de grande relevância um olhar e uma escuta a esses indivíduos também. 


\section{Família e paciente oncológico: uma atenção primária}

O processo maturativo biopsicossocial pelo qual o ser humano passa se dá em etapas evolutivas e peculiares, como a infância, a adolescência, a vida adulta e a terceira idade. Nessa trajetória, o indivíduo é influenciado pelas características socioculturais circunstantes, como também pelos vínculos familiares e afetivos, valores e crenças. 0 contexto em que ele está inserido exerce uma importante contribuição no seu desenvolvimento, visto que existe uma troca constante e entre indivíduo e meio social, podendo ser considerado então, um fator estruturante.

0 primeiro núcleo representativo do indivíduo é sua família, sendo essas pessoas muitas vezes a quem se busca semelhanças e exemplos para que, a partir de suas crenças e valores, o indivíduo possa realizar o processo de individuação e formação de sua personalidade. De acordo com essa concepção, a estrutura familiar tem influência dos tipos de relações e interações estabelecidas entre os membros, bem como do funcionamento dos membros da própria família (Minuchin \& Fishman, 1990 citados por Baptista, Baptista \& Dias, 2001).

Diante dessa perspectiva, considera-se a influência da família na construção do indivíduo como também no modo pelo qual ele se relacionará com os outros e as situações. Dentre estas situações, o adoecimento se apresenta como um evento existencial ameaçador para o paciente, mas que repercute também nos familiares no que concerne aos aspectos físicos, emocionais, econômicos e sociais.

Essas repercussões dos familiares ao vivenciarem a trajetória do adoecimento e a rotina de tratamento e cuidados junto com o paciente têm chances de interferir de forma favorável ou desfavorável no seu processo de enfrentamento. Apesar de a família muitas vezes ser sinônimo de suporte e rede de apoio, ela pode ser também um dificultador ao paciente dependendo de como seus membros experienciam as relações familiares, quais suas representações da doença e do tratamento e o impacto disso na dinâmica familiar.

Devido a isso, a família deve ser considerada também como uma unidade de cuidado da equipe multiprofissional, sendo essencial um olhar atento e um trabalho interdisciplinar com seus integrantes, visando a uma melhor adaptabilidade frente às situações vivenciadas e as relações humanas estabelecidas. Assim, "para além de intervenções específicas na depressão clínica é fundamental que exista um plano global e integral de cuidados, dirigido ao alívio da dor e de outros sintomas físicos, que responda as necessidades do doente e da família" (Trancas et al., 2010, pp. 1108-1109). A equipe então deve considerar a família como prestadora de cuidados, mas também incluir nos cuidados profissionais. A partir dessa perspectiva, o objeto de atenção e de cuidado da equipe deve ser o binômio paciente-família e ao considerar esta unidade, pode-se ter benefícios clínicos e inter-relacionais ao doente e sua família.

\section{Considerações finais}

No processo do adoecimento oncológico, tanto a mente quanto o corpo estão envolvidos, sendo necessária uma avaliação multiprofissional de fatores que abrangem a saúde geral do indivíduo, incluindo os aspectos físicos, psíquicos e socioeconômicos para que seja realizado um diagnóstico diferencial e oferecida uma terapêutica adequada ao sofrimento subjetivo do paciente e seus familiares. No que concerne à sintomatologia apresentada pelo paciente,

a diferença na avaliação da depressão está na linha tênue e imprecisa entre os quadros clínicos, subclínicos e não patológicos, condição que gera maior dificuldade diagnóstica pelo fato de os sintomas depressivos confundirem-se com os da doença clínica, como exemplo: fadiga, perda do apetite, alteração do sono, sintomas comuns desencadeados também pela quimioterapia (Gaspar, 2011, p. 109).

Tendo isso em vista, os profissionais se deparam com o desafio de avaliar uma dinâmica inter-relacionada de diversos fatores envolvidos, sendo importante então a atuação não apenas multiprofissional, mas principalmente interdisciplinar. Essa atuação em equipe tem como objetivo uma compreensão holística do indivíduo e suas relações, levando em consideração a experiência subjetiva. Essa proposta de trabalho, referida de intervenção psicossocial,

[...] contribui para o tratamento clínico atendendo às necessidades que surgem durante a trajetória do paciente oncológico: desde a situação do diagnóstico, o enfrentamento dos trata- 
mentos, à reabilitação e aos cuidados paliativos, em busca de melhor adaptabilidade do paciente ou da família aos desdobramentos com os quais deparam (Liberato \& Carvalho, 2008, p. 343).

A finalidade desta intervenção multi e interdisciplinar deve ser o cuidado e o acolhimento da vivência e sofrimento singular dos envolvidos, paciente e familiares, tendo como propósito oferecer recursos capazes de proporcionar uma melhor qualidade de vida, de acordo com suas possibilidades, desejos, crenças e valores. E quando os recursos são escassos ou insuficientes não significa que não tem mais o que fazer, pois "acompanhar a pessoa nessa busca pela melhora, a partir do referencial que ela tem de sua vida, já é uma maneira de oferecer cuidado" (Espinha \& Amatuzzi, 2008, p. 484).

A partir deste estudo, observa-se a necessidade de se considerar questões para além do âmbito físico que também interferem no adoecimento e, consequentemente, no tratamento e recuperação do paciente e suas relações familiares. Para isso, então, os profissionais de saúde devem ter desde a graduação conhecimento acerca das interferências entre as questões emocionais e físicas, bem como a implementação de discussão interdisciplinar de casos clínicos no ambiente de trabalho com o objetivo de verificar na prática essas interferências.

\section{Referências}

Baptista, M. N., Baptista, A. S. D., \& Dias, R. R. (2001). Estrutura e suporte familiar como fatores de risco na depressão de adolescentes. Psicologia Ciência e Profissão, 21(2). Recuperado em 19 dezembro, 2011, de http://pepsic.bvsalud.org

Baptista, M. N., Santos, K. M., \& Dias, R. R. (2006). Autoeficácia, lócus de controle e depressão em mulheres com câncer de mama. Revista Psicologia Argumento, 24(44), 27-36.

Bottino, S. M. B.; Fráguas, R., \& Gattaz, W. F. (2009) Depressão e câncer. Revista de Psiquiatria Clínica, 36(3). Recuperado em 15 dezembro, 2011, de http:// www.hcnet.usp.br/ipq/revista/vol36/s3/103.htm
Espinha, T. G., \& Amatuzzi, M. M. (2008). 0 cuidado e as vivências de internação em um hospital geral. Psicologia: Teoria e Pesquisa, 24(4). Recuperado em 19 dezembro, 2011, de http://www.scielo.br

Gaspar, K.C. (2011). Psicologia Hospitalar e Oncologia. In V. A. Angerami-Camon (Org.). Psicologia da Saúde: Um novo significado para a prática clínica (pp. 79126). São Paulo: Cengage Learning.

Graner, K. M., Cezar, L. T. S., \& Teng, C. T. (2008). Transtornos do humor em Psico-oncologia. In V. A. Carvalho (Org.). Temas em Psico-oncologia (pp. 243256). São Paulo: Summus.

Juliano, J. C. (1999). A arte de restaurar histórias: O diálogo criativo no caminho pessoal. São Paulo: Summus.

Juver, J. P. S., \& Verçosa, N. (2008) Depressão em pacientes com dor no câncer avançado. Revista Brasileira de Anestesiologia, 58(3). Recuperado em 19 de dezembro, 2011, de http://www.scielo.br

Liberato, R. P., \& Carvalho, V. A. (2008). Psicoterapia. In V. A. Carvalho (Org.). Temas em Psico-oncologia (pp. 341-350). São Paulo: Summus.

Peçanha, D. L. N. (2008). Câncer: recursos de enfrentamento na trajetória da doença. In V. A. Carvalho (Org.). Temas em Psico-oncologia (pp. 209-217). São Paulo: Summus.

Santos, C. B. (2004). Abordagem Centrada na Pessoa Relação Terapêutica e Processo de Mudança. Revista do Serviço de Psiquiatria do Hospital Fernando Fonseca. Recuperado em 22 de dezembro, 2011, de http://www.psilogos.com/Revista/Vol1N2/Indice2_ ficheiros/Santos.pdf

Teng, C. T., Humes, E. C., \& Demetrio, F. N. (2005). Depressão e comorbidades clínicas. Revista de Psiquiatria Clínica, 32(3). Recuperado em 15 de dezembro, 2011, de http://www.scielo.br/pdf/rpc/ v32n3/a07v32n3.pdf

Trancas, B., Cardoso, G., Luengo, A., Vieira, C., \& Reis, D. (2010). Depressão no doente oncológico: Considerações Diagnósticas e Terapêuticas. Revista Acta Médica Portuguesa, 23(6). Recuperado em 15 de dezembro, 2011, de http://www.actamedicaportuguesa.com/pdf/2010-23/6/1101-1112.pdf 